\title{
Análisis bibliométrico sobre la producción científica en distribución en planta en la red Redalyc durante el periodo 2007 - 2017
}

\author{
Bibliometric analysis on the scientific production in plant distribution in the \\ Redalyc network during the period 2007 - 2017
}

R. D. Buitrago-Pulido iD

\begin{abstract}
The distribution of the plant (layout), is a powerful strategy that brings with it the minimization of the total costs associated with the flow of raw materials and inputs in the organizations. Consequently, this research work aims to analyze the scientific production on Plant Distribution, using metrics to quantitatively show this production. To obtain data, the Redalyc search engine was used, which showed a sample of 79 articles, over a period of 10 years, from 2007 to 2017 . Once the information was tabulated, statistical analyzes were carried out using indicators bibliometric supported on 8 metrics. Finally, the results indicate that the university that has produced the most production on this subject is the Technological University of Pereira in its journal Scientia Et Technica. In the same way, these contributions were made in articles with a number of three authors. Likewise, Colombia is the largest producer of articles and were developed in 2014.
\end{abstract}

Index Terms - Bibliometry, Plant Distribution, Information Metrics, Scientific Production, Redalyc.

Resumen - La distribución de planta (Layout), es una poderosa estrategia que trae consigo la minimización de los costos totales asociados al flujo de materia prima e insumos en las organizaciones. En consecuencia, este trabajo de investigación tiene por objeto analizar la producción científica sobre Distribución en Planta, utilizando métricas que permitan mostrar cuantitativamente dicha producción. Para la obtención de datos, se utilizó el motor de búsqueda Redalyc, el cual arrojó una muestra de 79 artículos, en un periodo de 10 años, comprendido entre el 2007 al 2017. Una vez tabulada la información, se realizaron los análisis estadísticos utilizando indicadores bibliométricos apoyados en 8 métricas. Finalmente, los resultados indican que la universidad que mayor producción ha desarrollado en este tema es la Universidad Tecnológica de Pereira en su revista Scientia Et Technica. De la misma manera, estas contribuciones fueron realizadas en artículos con un número de tres autores. Así mismo, Colombia es el país mayor productor de artículos y fueron desarrolladas en el año 2014.

Palabras clave - Bibliometría, Distribución en Planta, Métricas de Información, Producción Científica, Redalyc

Este manuscrito fue enviado el 20 de abril de 2019 y aceptado el 23 de septiembre de 2019.

\section{INTRODUCTION}

$\mathrm{L}$ A distribución de planta es una metodología para realizar un ordenamiento en sistemas de manufactura, basados en métodos cuantitativos y técnicas multicriterio. Esta técnica ha sido usada en distintos sectores económicos, con el objeto de obtener diferentes rutas para el flujo del producto, mejoras en los tiempos de producción, la productividad y la eficiencia [1].

Dada la importancia de la premisa anterior, es relevante realizar una revisión de la producción científica acerca de la distribución en planta, con el objeto de analizarlo biométricamente.

El análisis Bibliométrico de las publicaciones científicas constituye una parte fundamental dentro de las herramientas del proceso de investigación, se ha convertido en un método de evaluación que permite calificar el proceso de generar conocimiento y su impacto en el entorno científico [2]. Los indicadores Bibliométricos resultan los parámetros más idóneos para valorar la actividad científica de un autor, de un grupo de trabajo, de una revista o de un país. No sólo permiten analizar la actividad científica en un momento determinado, sino también su evolución [3].

Prácticamente toda la literatura científica publicada en revistas contiene citas o referencias bibliográficas que proporcionan los precedentes sobre lo que el autor desea exponer en su trabajo, por ello constituyen una fuente de datos que permite conocer qué información consumen los autores [4]. Si bien el análisis de las referencias, como área de la Bibliometría, está sujeto a múltiples controversias y limitaciones, se acepta como una metodología adecuada para la evaluación de la producción científica basándose en el criterio que si un documento está mencionado en la lista de referencias de un artículo supone una relación entre el documento que se escribe y el referenciado [5].

R. D. Buitrago-Pulido, docente asociado de la dirección de Ingeniería Industrial la Universidad ECCI (Grupo de Investigación INDETECA). Bogotá, Colombia (E-mail: rbuitragop@ecci.edu.co) 
La Bibliometría es la aplicación de las matemáticas y métodos estadísticos a toda fuente escrita que esté basada en las facetas de la comunicación y que considere los elementos tales como autores, título de la publicación, tipo de documento, idioma, resumen y palabras claves o descriptores.

En [6] se puede identificar que hacia 1969 se proporcionó la primer definición formal de bibliometrics (Bibliometría), cuyo concepto propone que la aplicación de los métodos estadísticos y matemáticos para definir los procesos de la comunicación escrita, la naturaleza y el desarrollo de las disciplinas científicas mediante técnicas de recuento y análisis de la comunicación, es bibliometría.

Otro concepto fue desarrollado en [7], cuya perspectiva del constructo es direccionado hacia la técnica que tiene por objeto calcular la extensión o medida de los libros tomando como base diversos coeficientes, formato, tipo de letra, cantidad de palabras, peso del papel, etc.

Así mismo [8] afirma que la bibliometría es la aplicación de las matemáticas y los métodos estadísticos para analizar el curso de la comunicación escrita y de una disciplina. Dicho de otra manera, es la aplicación de tratamientos cuantitativos a las propiedades del discurso escrito y sus comportamientos típicos.

Desde otra perspectiva, [9] identifica la bibliometría como una herramienta metodológica que parte de la necesidad de cuantificar ciertos aspectos de la ciencia y que una de las facetas de la cienciometría sería la Bibliometría, entendida como el cómputo de diversos indicadores de publicaciones que los científicos producen.

El primer estudio Bibliométrico pareciera ser el de Colé y Eales (1917). Ellos realizaron un análisis estadístico de las publicaciones sobre anatomía comparativa entre 1550 y 1860 con la distribución por países y las divisiones del reino animal [10]. Posteriormente, E. Hulme (1923), bibliotecario de la British Patent Office, presenta un análisis estadístico de la historia de la ciencia.

En (1927), Gross y Gross analizaron las referencias hechas en artículos de revistas sobre química indizadas en The Journal of the American Chemistry Society para 1926. Este es el primer trabajo registrado de cuenta y análisis de citaciones [10].

\section{METODOLOGÍA}

Para cumplir los objetivos de esta investigación, es necesario usar un enfoque cuantitativo-descriptivo de tipo transversal [11], y a su vez retrospectivo [4], de tal forma que sea posible la selección y organización de los documentos. La unidad de análisis es la palabra "Distribución en Planta" aplicada a la Ingeniería Industrial, y el periodo de observación es 2007 a 2017 en las métricas productividad autoral, distribución de contribuciones de autoría, productividad de artículos por revista, productividad idiomática, productividad de artículos por año, productividad de artículos por país, productividad de artículos por institución y la concurrencia de las palabras clave de los artículos publicados.

La muestra estaba conformada por 79 documentos publicados en el periodo 2007 - 2017 en la base de datos Redalyc en los idiomas español e inglés. Los criterios de búsqueda fueron condicionados mediante el uso de lógica Booleana.

La búsqueda en inglés se llevó a cabo mediante la ecuación ("plant distribution" OR layout AND industry NOT Biology) OR ("plant design" AND industry NOT Biology) OR ("Location of facilities" AND industriy NOT Biology). En español la ecuación quedó configurada de la siguiente manera, ("Distribución en planta" AND Industria NOT Biología) OR ("Diseño de planta" AND Industria NOT Biología) OR ("Localización de instalaciones" AND Industria NOT Biología).

Tras aplicar las ecuaciones correspondientes se obtuvo una muestra de 376 artículos, y posteriormente se aplicaron criterios de inclusión y exclusión basados en fecha (2007 - 2017), Peerreviewed (Documentos que hayan sido objeto de un proceso de revisión por pares). Como consecuencia de la aplicación de los criterios mencionados se obtuvo una muestra final de 79 artículos. Cabe mencionar que Redalyc no permite hacer un filtro por límite geográfico, sin embargo, de forma indexada este está aplicado por la naturaleza de la base de datos, ya que las contribuciones provienen de diversas fuentes Latinoamericanas.

Una vez obtenida la muestra se pasó a examinar cada uno de ellos. A continuación, se describen los pasos utilizados según la metodología propuesta en [12], a) Seleccionar los documentos, y b) Identificar las variables para caracterizar la información.

\section{RESULTADOS Y DISCUSIÓN}

La información que se presenta a continuación proviene de 79 artículos encontrados en la Red de Revistas Científicas de América Latina y el Caribe, España y Portugal REDALYC.

La tabla I muestra que el $34 \%$ de las contribuciones de autoría corresponden a publicaciones desarrollas por 3 autores, seguido por el $27 \%$ equivalente a 21 artículos escritos por 2 autores y el $20 \%$ correspondiente a 16 escritos por 1 autor y el $19 \%$ restante que corresponden a 15 artículos de la muestra, que fueron escritos por 4 y 5 autores.

TABLA I DISTRIBUCIÓN DE CONTRIBUCIONES DE AUTORÍA

\begin{tabular}{ccc}
\hline \hline $\mathrm{N}^{\circ}$ de artículos & $\mathrm{N}^{\circ}$ de autores & $\%$ de participación \\
\hline 3 & 5 & $4 \%$ \\
12 & 4 & $15 \%$ \\
16 & 1 & $20 \%$ \\
21 & 2 & $27 \%$ \\
27 & 3 & $34 \%$ \\
\hline \hline
\end{tabular}


La tabla II permite evidenciar que 13 artículos correspondientes al $16 \%$, fueron publicados en la revista Scientia Et Technica de la Universidad Tecnológica de Pereira - Colombia, 8 artículos correspondientes al 10\%, fueron publicados en la revista Industrial Data de la Universidad Nacional Mayor de San Marcos - Perú, 7 artículos correspondientes al 9\%, fueron publicados en la revista Ingeniería Industrial, Actualidad y Nuevas Tendencias de la Universidad de Carabobo - Venezuela, 6 artículos correspondientes a un $8 \%$, se publicaron en la revista Ingeniería Industrial del Instituto Superior Politécnico José Antonio Echeverría - Cuba, 4 artículos correspondientes al 5\%, fueron publicados por la revista Tecnura de la Universidad Distrital Francisco José de Caldas - Colombia y el resto correspondiente a 40 artículos sumando un 52\%, fueron publicados por revistas de Chile, Colombia, Cuba y México con un número de publicaciones entre 1 y 4 artículos y un porcentaje de participación también entre el $1 \%$ y el $4 \%$.

TABLA II PRODUCTIVIDAD DE ARTÍCULOS POR REVISTA

\begin{tabular}{lcc}
\hline \multicolumn{1}{c}{ Revista } & $\mathrm{N}^{\circ}$ de artículos & $\%$ de participación \\
\hline Scientia Et Technica & 13 & $16 \%$ \\
Industrial Data & 8 & $10 \%$ \\
Ingeniería Industrial. & & \\
Actualidad y Nuevas & 7 & $9 \%$ \\
Tendencias & 6 & \\
Ingeniería Industrial & 4 & $8 \%$ \\
Tecnura & 40 & $5 \%$ \\
Otras &
\end{tabular}

En la contribución por idioma de la tabla III, se puede evidenciar que 77 artículos de la muestra, correspondientes al $97 \%$, fueron escritos en español y 2 artículos en idiomas como el inglés y portugués.

TABLA III

PRODUCTIVIDAD IDIOMÁTICA

\begin{tabular}{lcc}
\hline \hline Idioma & $\mathrm{N}^{\circ}$ de artículos & \% de participación \\
\hline Español & 77 & $97 \%$ \\
Otros idiomas & 2 & $3 \%$ \\
\hline \hline
\end{tabular}

En la tabla IV es posible validar que el mayor número de artículos fue escrito en el 2014 con 14 artículos correspondientes al 18\%, seguido del 2007 con 10 artículos correspondientes al 13\%, continuando con el 2013 con 8 artículos correspondientes al 10\%, 2011, 2015 y 2016 cada uno con 7 artículos equivalentes al $9 \%$ y el resto de los 24 artículos correspondientes al 30\% fueron escritos entre el 2008, 2009, 2010, 2012 y 2017 sumando a esta cifra 2 artículos que se encuentran fuera de red correspondientes al $3 \%$.

\begin{tabular}{|c|c|c|}
\hline Años & $\mathrm{N}^{\circ}$ de artículos & $\%$ de participación \\
\hline 2014 & 14 & $18 \%$ \\
\hline 2007 & 10 & $13 \%$ \\
\hline 2013 & 8 & $10 \%$ \\
\hline 2016 & 7 & $9 \%$ \\
\hline 2015 & 7 & $9 \%$ \\
\hline 2011 & 7 & $9 \%$ \\
\hline 2010 & 6 & $8 \%$ \\
\hline 2009 & 6 & $8 \%$ \\
\hline 2008 & 5 & $6 \%$ \\
\hline 2012 & 5 & $6 \%$ \\
\hline 2017 & 2 & $3 \%$ \\
\hline Artículo Fuera de Línea & 2 & $3 \%$ \\
\hline
\end{tabular}

Como se puede observar en la tabla $\mathrm{V}$, el mayor número de artículos fue escrito en Colombia con un $48 \%$ equivalente a 38 artículos, seguida por Cuba con un $14 \%$ correspondiente a 11 artículos, Perú con un $10 \%$ equivalente a 8 artículos, México y Venezuela con un $9 \%$ que suman 7 artículos cada uno, Chile con un $8 \%$ equivalente a 6 artículos, y finalmente con 2 artículos de los 79 que se encontraban fuera de red al momento de la consulta.

TABLA V PRODUCTIVIDAD DE ARTÍCULOS POR PAÍS

\begin{tabular}{lcc}
\hline \multicolumn{1}{c}{ Países } & $\mathrm{N}^{\circ}$ de artículos & \% de participación \\
& & \\
\hline Colombia & 38 & $48 \%$ \\
Cuba & 11 & $14 \%$ \\
Perú & 8 & $10 \%$ \\
Venezuela & 7 & $9 \%$ \\
México & 7 & $9 \%$ \\
Chile & 6 & $8 \%$ \\
Artículo Fuera de Línea & 2 & $3 \%$ \\
\hline \hline
\end{tabular}

La métrica productividad de artículos en Colombia contenido en la tabla VI, presenta 11 instituciones que han aportado 38 artículos de las cuales su mayor representante es la Universidad Tecnológica de Pereira con 11 artículos correspondientes al $29 \%$, seguida de la Universidad Nacional de Colombia con 6 artículos equivalentes al $16 \%$, la Universidad Distrital Francisco José de Caldas con 4 artículos que suman un $11 \%$ de toda la productividad, la Universidad ICESI, la Escuela de Ingeniería de Antioquia, la Universidad Militar Nueva Granada y Universidad del Valle cada una con un aporte de 3 artículos que suman un 32\% acumulado, la Universidad de Medellín con 2 artículos correspondientes al 5\% y finalmente con 1 artículo la Pontificia Universidad Javeriana, la Universidad de Antioquia y la Universidad del Norte que hacen un $3 \%$ cada una.
TABLA IV

PRODUCTIVIDAD DE ARTÍCULOS POR AÑO
TABLA VI

PRODUCTIVIDAD DE ARTÍCULOS EN COLOMBIA 


\begin{tabular}{lcc}
\hline \multicolumn{1}{c}{ Paises } & $\mathrm{N}^{\circ}$ de artículos & \% de participación \\
\hline $\begin{array}{l}\text { Universidad Tecnológica de } \\
\text { Pereira }\end{array}$ & 11 & $29 \%$ \\
$\begin{array}{l}\text { Universidad Nacional de } \\
\text { Colombia }\end{array}$ & 6 & $16 \%$ \\
Universidad Distrital & & \\
Francisco José de Caldas & 4 & $11 \%$ \\
$\begin{array}{l}\text { Universidad ICESI } \\
\text { Escuela de Ingeniería de } \\
\text { Antioquia }\end{array}$ & 3 & $8 \%$ \\
$\begin{array}{l}\text { Universidad Militar Nueva } \\
\text { Granada }\end{array}$ & 3 & $8 \%$ \\
$\begin{array}{l}\text { Universidad del Valle } \\
\text { Universidad de Medellín }\end{array}$ & 3 & $8 \%$ \\
$\begin{array}{l}\text { Pontificia Universidad } \\
\text { Javeriana } \\
\text { Universidad de Antioquia }\end{array}$ & 3 & $8 \%$ \\
Universidad del Norte & 1 & $5 \%$ \\
\hline \hline
\end{tabular}

La tabla VII permite evidenciar que 11 de los 79 artículos fueron escritos por la Universidad Tecnológica de Pereira Colombia, 8 de los artículos fueron escritos por el Instituto Superior Politécnico José Antonio Echeverría - Cuba y la Universidad Nacional Mayor de San Marcos - Perú cada uno, 7 de los artículos fueron escritos por la Universidad de Carabobo - Venezuela, 6 por la Universidad Nacional de Colombia, 4 por la Universidad Distrital Francisco José de Caldas y el resto de instituciones tienen entre 1 y 3 artículos cada una que suman el $44 \%$ acumulado para un total de 35 artículos.

TABLA VII

\begin{tabular}{lcc}
\multicolumn{1}{c}{ PRODUCTIVIDAD DE ARTÍCULOS POR INSTITUCIÓN } \\
\hline \multicolumn{1}{c}{ Institución } & $N^{\circ}$ de artículos & $\%$ de participación \\
\hline $\begin{array}{l}\text { Universidad Tecnológica } \\
\text { de Pereira }\end{array}$ & 11 & $14 \%$ \\
$\begin{array}{l}\text { Universidad Nacional } \\
\text { Mayor de San Marcos }\end{array}$ & 8 & $10 \%$ \\
$\begin{array}{l}\text { Instituto Superior } \\
\text { Politécnico José Antonio } \\
\text { Echeverría }\end{array}$ & 8 & $10 \%$ \\
Universidad de Carabobo & 7 & $9 \%$ \\
$\begin{array}{l}\text { Universidad Nacional de } \\
\text { Colombia }\end{array}$ & 6 & $8 \%$ \\
Universidad Distrital \\
Francisco José de Caldas
\end{tabular}

El análisis de concurrencia mostrado en la tabla VIII, refleja que hay una convergencia en 77 palabras clave de los artículos extraídos de la muestra de Redalyc, en donde el 5\% corresponde a las palabras Distribución en Planta y Distribución de Planta, con una frecuencia de 4, el 3\% para Manufactura esbelta con frecuencia igual a 3, y Localización de Instalaciones junto con Algoritmo de búsqueda Tabú con el $2 \%$. Otros términos corresponden al $76 \%$ con una concurrencia de 1 vez.

TABLA VIII

CONCURRENCIA DE LAS PALABRAS CLAVE

\begin{tabular}{|c|c|c|}
\hline Palabras & Concurrencia & $\%$ de participación \\
\hline $\begin{array}{l}\text { Distribución de } \\
\text { planta }\end{array}$ & 4 & $5 \%$ \\
\hline $\begin{array}{l}\text { Distribución en } \\
\text { planta }\end{array}$ & 4 & $5 \%$ \\
\hline $\begin{array}{l}\text { Manufactura } \\
\text { esbelta }\end{array}$ & 3 & $4 \%$ \\
\hline $\begin{array}{l}\text { Localización de } \\
\text { instalaciones }\end{array}$ & 2 & $3 \%$ \\
\hline $\begin{array}{l}\text { Algoritmo de } \\
\text { Búsqueda Tabú }\end{array}$ & 2 & $3 \%$ \\
\hline $\begin{array}{l}\text { Celdas de } \\
\text { manufactura }\end{array}$ & 1 & $1 \%$ \\
\hline $\begin{array}{l}\text { Programación } \\
\text { lineal }\end{array}$ & 1 & $1 \%$ \\
\hline $\begin{array}{l}\text { Proceso } \\
\text { productivo }\end{array}$ & 1 & $1 \%$ \\
\hline $\begin{array}{l}\text { Diagnóstico } \\
\text { ergonómico }\end{array}$ & 1 & $1 \%$ \\
\hline $\begin{array}{l}\text { Demanda } \\
\text { energética }\end{array}$ & 1 & $1 \%$ \\
\hline Diseño de planta & 1 & $1 \%$ \\
\hline $\begin{array}{l}\text { Comportamient } \\
\text { o sísmico }\end{array}$ & 1 & $1 \%$ \\
\hline $\begin{array}{l}\text { Sociedad } \\
\text { globalizada }\end{array}$ & 1 & $1 \%$ \\
\hline $\begin{array}{l}\text { Producción y } \\
\text { transporte }\end{array}$ & 1 & $1 \%$ \\
\hline $\begin{array}{l}\text { Algoritmos } \\
\text { Evolutivos }\end{array}$ & 1 & $1 \%$ \\
\hline $\begin{array}{l}\text { Recursos } \\
\text { naturales }\end{array}$ & 1 & $1 \%$ \\
\hline $\begin{array}{l}\text { Cadena de } \\
\text { suministros }\end{array}$ & 1 & $1 \%$ \\
\hline $\begin{array}{l}\text { Capacidad de } \\
\text { producción }\end{array}$ & 1 & $1 \%$ \\
\hline Diseño óptimo & 1 & $1 \%$ \\
\hline Otras & 48 & $62 \%$ \\
\hline
\end{tabular}

\section{CONCLUSIONES}

En el desarrollo de esta investigación, se ha analizado la producción científica sobre Distribución de Planta. El primer indicador Bibliométrico, contribuciones por autoría, es determinante para afirmar que los artículos fueron desarrollados en colaboración, y el número representativo de integrantes es igual a tres. Las razones que justifican el dato obtenido atienden a criterios de limitación en número participantes en: a) convocatorias de revistas, b) convocatorias para financiar 
proyectos de investigación internos y externos y, c) Convocatorias en eventos científicos.

En cuanto a la productividad de artículos por revista, Scientia Et Technica de la Universidad Tecnológica de Pereira Colombia, es la revista que más publicaciones ha realizado sobre Distribución en Planta. Lo anterior responde a diversas situaciones, en donde es relevante destacar que la Universidad cuenta con un programa de doctorado en ingeniería cuya línea de investigación tiene énfasis en Industria, Sistemas Sociales y Modelamiento, lo cual permite un sostenimiento de producción científica constante en distribución en planta, rama de la investigación de operaciones. En adición a lo anterior, la facultad de Ingeniería Industrial de esta misma institución, tiene un programa de maestría en Investigación de Operaciones y Estadística, el cual desarrolla proyectos de investigación aplicados al contexto industrial dentro del tema distribución en planta. Lo anterior fue constatado en los artículos que comprenden la muestra objeto de análisis de esta investigación.

Con respecto a la productividad idiomática setenta y siete artículos fueron escritos en el idioma español, los dos (2) artículos restantes se encuentran en inglés y portugués. En concordancia con la productividad por revista, este aporte fue llevado a cabo en Colombia en revistas que no exigían otro idioma para sus contribuciones. Cabe precisar que Redalyc difunde producción científica de Iberoamérica, por tanto, los cientos de instituciones de educación superior, centros de investigación, asociaciones profesionales y editoriales promueven la publicación en español.

En cuanto a la concurrencia, la palabra clave que más frecuencia tiene para la caracterización del tema de los manuscritos es distribución de planta. Es de acotar que este término fue buscado en Tesauros en español, UNESCO, Buscador del ITESO, y los términos asociados son planeación de las máquinas y planeación de la industria.

Finalmente, los resultados de esta investigación conducen a reflexionar en la ampliación de los propósitos de futuros trabajos en este campo, en donde el espectro debe extenderse a bases de datos como WoS y SCOPUS, de tal forma que no se tenga límite geográfico a causa del origen de las contribuciones, y pueda evaluarse los índices de calidad h y su SJR.

\section{REFERENCIAS}

C. Pantoja, J. P. Orejuela, and J. J. Bravo, "Metodología de distribución de plantas en ambientes de agrupación celular," Estud. Gerenciales, vol. 33, no. 143, pp. 132-140, Apr. 2017.

[2] C. F. Rueda-Clausen, C. Villa-Roel Gutíerrez, and C. E. RuedaClausen, "Indicadores bibliométricos: origen, aplicación, contradicción y nuevas propuestas," MedUNAB, vol. 8, no. 1, p. 8, 2005.

[3] Ferrand, P. Odou, D. Lebel, and J. F. Bussières, "Evaluation of knowledge and perceptions of pharmacists and residents pharmacists about bibliometric indicators applied to the scientific literature in pharmacy," Annales Pharmaceutiques Francaises, vol. 77, no. 3. Elsevier Masson, pp. 250-263, 01-May-2019. "Bibliometric analysis of global Lassa fever research (1970-2017): a 47 - year study," BMC Infect. Dis., vol. 18, no. 1, p. 639, Dec.

[5] R. Mutz and H. D. Daniel, "The bibliometric quotient (BQ), or how to measure a researcher's performance capacity: A Bayesian Poisson Rasch model," J. Informetr., vol. 12, no. 4, pp. 1282-1295, Nov. 2018

[6] L. Leydesdorff, P. Wouters, and L. Bornmann, "Professional and citizen bibliometrics: complementarities and ambivalences in the development and use of indicators-a state-of-the-art report," Scientometrics, vol. 109, no. 3, pp. 2129-2150, Dec. 2016.

[7] G. Lewison, S. Kumar, C. Y. Wong, P. Roe, and R. Webber, "The contribution of ethnic groups to Malaysian scientific output, 19822014 , and the effects of the new economic policy," Scientometrics, vol. 109, no. 3, pp. 1877-1893, Dec. 2016.

[8] K. Barker, "The UK research assessment exercise: The evolution of a national research evaluation system," Res. Eval., vol. 16, no. 1, pp. 3-12, Mar. 2007.

[9] E. Jiménez-Contreras, N. Robinson-García, and Á. Cabezas-Clavijo, "Productividad e impacto de los investigadores españoles: umbrales de referencia por áreas científicas," Rev. española Doc. Científica, vol. 34, no. 4, pp. 505-525, Dec. 2011.

[10] L. Michán and I. Muñoz-Velasco, "Cienciometría para ciencias médicas: definiciones, aplicaciones y perspectivas," Investig. en Educ. Médica, vol. 2, no. 6, pp. 100-106, Apr. 2015.

[11] R. Hernández Sampieri, Metodología de la investigación. 2014.

[12] G. Lorenzo Lledó and C. Scagliarini Galiano, "Revisión bibliométrica sobre la realidad aumentada en Educación," Rev. Gen. Inf. y Doc., vol. 28, no. 1, pp. 45-60, Jul. 2018.

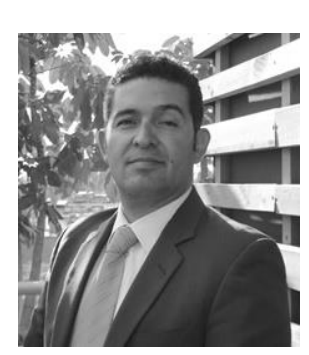

R. D. Buitrago-Pulido, nació el 30 de enero de 1980 en Bogotá, Colombia. $\mathrm{PhD}$ Candidate de la Universitat Illes Balears en Tecnología Educativa, recibió título de Magister en Tecnologías de la Información Aplicadas a la Educación en la UPNColombia en 2014. Especialista en Gerencia de Mantenimiento de la Universidad ECCI en 2011, y es profesional en Ingeniería Mecánica egresado de la Universidad Central de Colombia del año 2004. Desarrolla investigación en el campo de la Mixed Reality aplicada a la optimización de procesos industriales y a la educación. Actualmente es docente asociado de la Universidad ECCI y pertenece al grupo de investigación INDETECA.

ORCID: http://orcid.org/0000-0002-4893-9880 